\title{
Fully porous GaN p-n junction diodes fabricated by chemical vapor deposition
}

\author{
O.V. Bilousov ${ }^{\mathrm{a}}$, J.J. Carvajal ${ }^{\mathrm{a}, *}$, H. Geaney ${ }^{\mathrm{b}, \mathrm{c}}$, V. Z. Zubialevich ${ }^{\mathrm{c}}$, P.J. Parbrook ${ }^{\mathrm{c}, \mathrm{d}}$, O. \\ Martínez $^{\mathrm{e}}$, J. Jiménez ${ }^{\mathrm{e}}$, F. Díaz ${ }^{\mathrm{a}}$, M. Aguilóa ${ }^{\text {, C. O’Dwyer }}{ }^{\mathrm{b}, \mathrm{c}, *}$ \\ ${ }^{a}$ Física i Cristal·lografia de Materials i Nanomaterials (FiCMA-FiCNA) and EMaS, \\ Universitat Rovira i Virigli (URV), Marcel·lí Domingo s/n, E-43007 Spain \\ ${ }^{\mathrm{b}}$ Department of Chemistry, University College Cork, Cork, Ireland \\ c Tyndall National Institute, Lee Maltings, Dyke Parade, Cork, Ireland \\ d School of Engineering, University College Cork, Cork, Ireland \\ e GdS-Optronlab, Departamento Física Materia Condensada, Univ. de Valladolid, \\ Edificio I+D, Paseo de Belén, 11, 47011, Valladolid, Spain \\ *corresponding authors: joanjosep.carvajal@urv.cat, c.odwyer@ucc.ie
}

\section{Abstract}

\section{Introduction}

Modern society is experiencing an ever-increasing demand for energy to power a vast array of electrical and mechanical devices. A significant amount of the energy consumed is used for lighting purposes. For instance, this demand is $\sim 17 \%$ of the total energy consumed in the USA in 2011 [1]. Thus, any approach that can reduce energy consumption is important. In this context, the development of light emitting diodes (LEDs) incorporating at least one porous component, with improved light extraction efficiency, is being explored intensively [2]. However, up to now, only partially porous $p-n$ junctions have been analyzed for this purpose.

Despite the wide interest of porous semiconductors in view to their peculiar electrical, optical, and mechanical properties for applications in many fields, from electronics to optoelectronics [3], from photovoltaics [4] to biomedicine and environment monitoring [5], fabrication of totally porous $p-n$ junctions has been scarcely investigated and restricted almost exclusively to porous Si or III-Vs produced by anodization. The rectifying behavior of entirely porous $p$ - $n$ junctions is, from a theoretical point of view, compatible with that of a high-density of randomly distributed elemental mesoscopic $p$ - $n$ crystalline junctions operating in parallel [6]. Also, it has been pointed out that conventional planar non-porous diode structures exhibit a number of inherent deficiencies that result in relatively low energy-conversion efficiencies, a problem that might be overcome by completely porous $p-n$ junctions [7]. In fact, a tenfold enhancement of efficiency compared to that of the usual planar non-porous devices has been demonstrated for totally porous Si $p$ - $n$ junctions in betavoltaics, and it has been postulated that significant efficiency gain might be expected also in photo detectors and solar cells fabricated using completely porous $p$ - $n$ junctions [7].

The use of fully porous $p-n$ junctions might be especially important for enhancing the light extraction of LEDs. It has been demonstrated that the induction of a porous structure in the LED device can lead to an enhancement of the light extraction efficiency from the device [2a]. In fact, the induction of partially porous $p$ - $n$ junctions in GaN-based LEDs has lead to different improvements of the light extraction efficiencies. 
For instance, an increase of $12 \%$ of the internal quantum efficiency and $23 \%$ higher optical power has been reported on a GaN diode including a photonic crystal structure in its $n$-type component [2f], while an increase by $70 \%$ on the optical output power has been demonstrated for a LED with a photonic crystal structure selectively grown on $p$ type GaN [2i]. Using nanotextured $p$-type $\mathrm{GaN}, 46 \%$ higher light output than in a standard LED with unpatterned $p$-type GaN has been reported [2j]. Furthermore, it has been postulated that if the porous structure, forming a photonic crystal structure, crosses the active region of the LED, i.e. the $p$ - $n$ junction thus forming an entirely porous $p-n$ junction, the increase in light extraction efficiency could be enhanced even further [2b]. A light output power improvement as high as $94 \%$ has been demonstrated for the structures fabricated by chemical etching, in which porosity affected even the ITO electrode [2k].

Recently, we have demonstrated that it is possible to produce nanoporous GaN by crystal growth methods during chemical vapor deposition (CVD), and without the need of any post-growth treatment to induce porosity [8]. We have also demonstrated that we can produce both $n$-type and $p$-type porous $\mathrm{GaN}$ by this methodology with high density of charge carriers $\left(\sim 10^{18} \mathrm{~cm}^{-3}\right)$ and even forming low resistivity Ohmic contacts with high work function metals such as Au or Pt [9].

In this paper, we present the fabrication of fully porous $p$ - $n$ junctions by using nanoporous GaN produced by CVD, and we compare their performance with partially porous $p$ - $n$ junctions fabricated using the same methodology, in which only the $n$-type or $p$-type $\mathrm{GaN}$ layers are porous. We characterized their diode behavior at high temperatures, and demonstrated their ability as current rectifiers, thus proving the potential of these fully porous $p-n$ junctions for diode and LEDs applications.

Epitaxial nanoporous GaN thin layers were grown by CVD by the direct reaction of metallic Ga with ammonia in a tubular reactor on non-porous GaN (0001) thin films

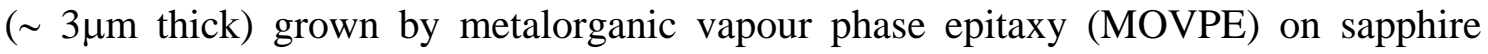
(0001) substrates. After degassing the quartz reactor to a vacuum pressure of $1 \times 10^{-2}$ Torr, ammonia was introduced through a mass-flow controller at a flow rate of $75 \mathrm{sccm}$, while the furnace was heated to $1200 \mathrm{~K}$. The growth lasted for $30 \mathrm{~min}$ at the constant flow of $\mathrm{NH}_{3}$ and temperature. After growth, the furnace was cooled down to room temperature and the $\mathrm{NH}_{3}$ flow was stopped, reducing the pressure inside the reactor to 1 $\times 10^{-2}$ Torr. Figure 1 shows an schematic description of the process. As a substrate we used non-porous GaN (0001) thin films deposited on sapphire (0001) either $n$-type or $p$ type wafers, depending on the experiment and the $p$ - $n$ junction to be formed (Fig. 1a,b). Mg doped samples were grown to produce $p$-type nanoporous GaN films (Fig. 1b). These samples were post-growth annealed in a $\mathrm{N}_{2}$ atmosphere at $973 \mathrm{~K}$ for 20 min in order to break the existing Mg-H complexes and activate the $p$-type conductivity of the porous GaN layers [10]. Fully porous $p-n$ junctions were fabricated in a two crystal growth step process (Fig. 1c). First, an undoped $n$-type porous GaN layer was grown on non-porous $\mathrm{GaN}(0001)$. Second, another porous Mg-doped p-type $\mathrm{GaN}$ layer was grown on the top of the porous $n$-type GaN layer as represented in Figure 1, under the same reaction conditions. 

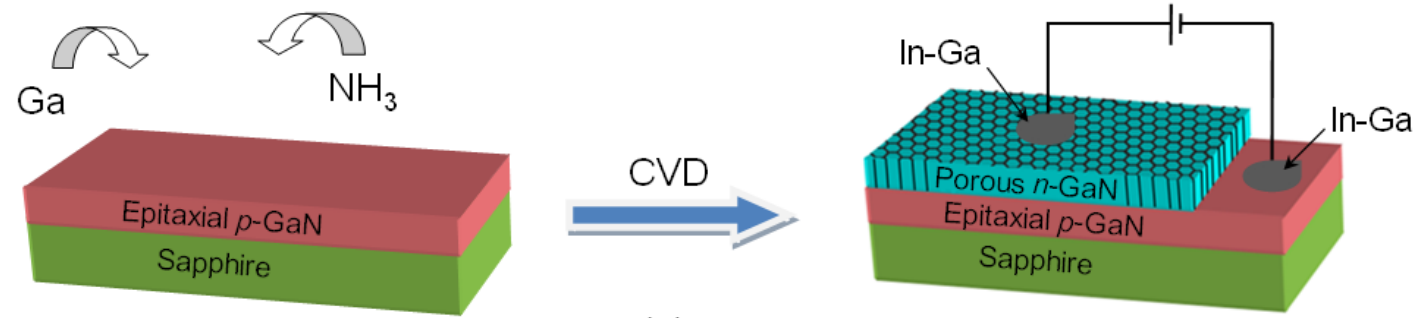

(a)
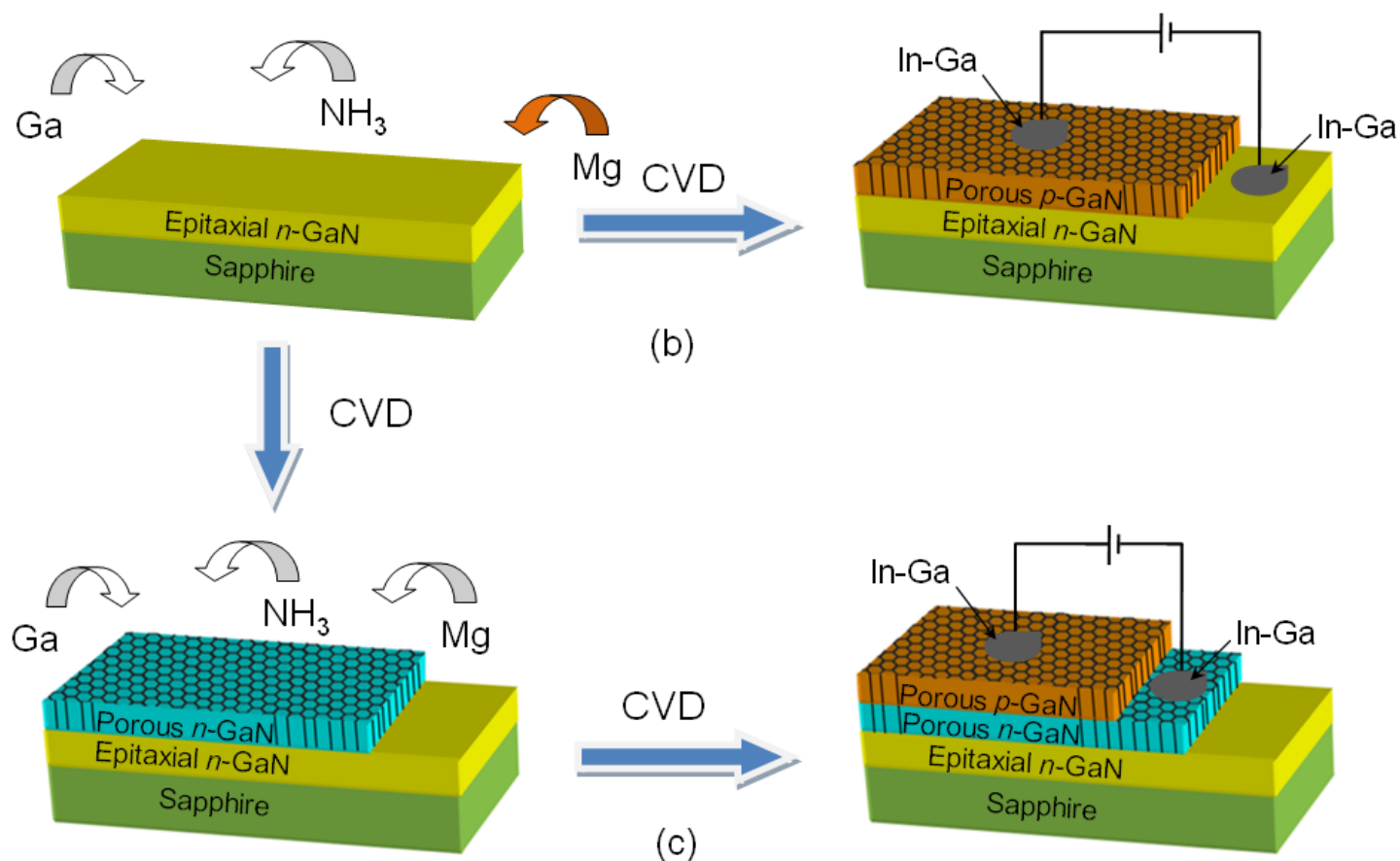

(b)

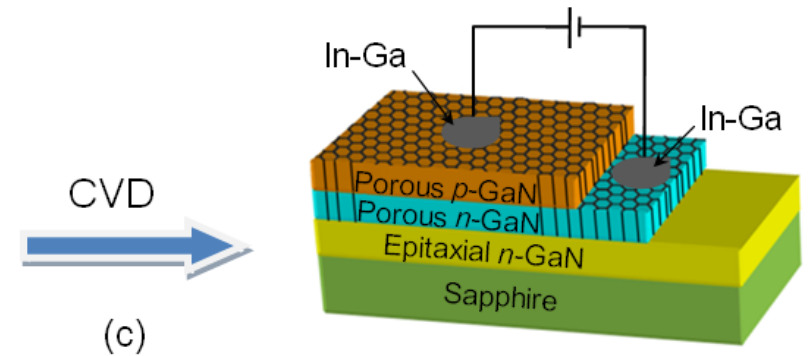

Figure 1. Schematic representation of the formation process of partially and fully porous GaN $p-n$ junctions by CVD.

In this way, three types of porous $\mathrm{GaN}$ diodes were prepared: (i) undoped $n$-type porous GaN on non-porous $p$-type GaN (hereafter porous $n$ - $p$ diode), Figure 2(a); Mgdoped porous $p$-type GaN on non-porous $n$-type $\mathrm{GaN}$ (hereafter porous $p$ - $n$ diode), Figure 2(b); and Mg-doped porous GaN on undoped porous $n$-type GaN (hereafter fully porous $p$ - $n$ diode), Figure 2(c).

Figure 2 shows also top-view SEM images of the porous GaN layers. All porous $\mathrm{GaN}$ layers reveal a high degree of porosity with a large number of pores. Despite being grown under the same conditions, the porous $p$-type layers tend to show larger diameter pores when compared to those observed on the $n$-type porous layers. This is especially significant for fully porous GaN $p$ - $n$ diodes. The pores observed in the $p$-type layer are significantly wider than those on the $n$-type layer prior to the second growth step that might be related to the corrugation of pores due to a lower decomposition temperature of Mg-doped GaN [11], which would be accentuated by the presence of the pores. High magnification images of the porous structures for $n$-type and $p$-type GaN layers can be seen in Figure S1 of the Supporting Information.

In all cases, however, the porous GaN layers are grown oriented along the $\boldsymbol{c}$ crystallographic direction, matching the crystallographic orientation of the non-porous epitaxial GaN thin-films used as substrates. Pore hollows are always parallel to this direction. 

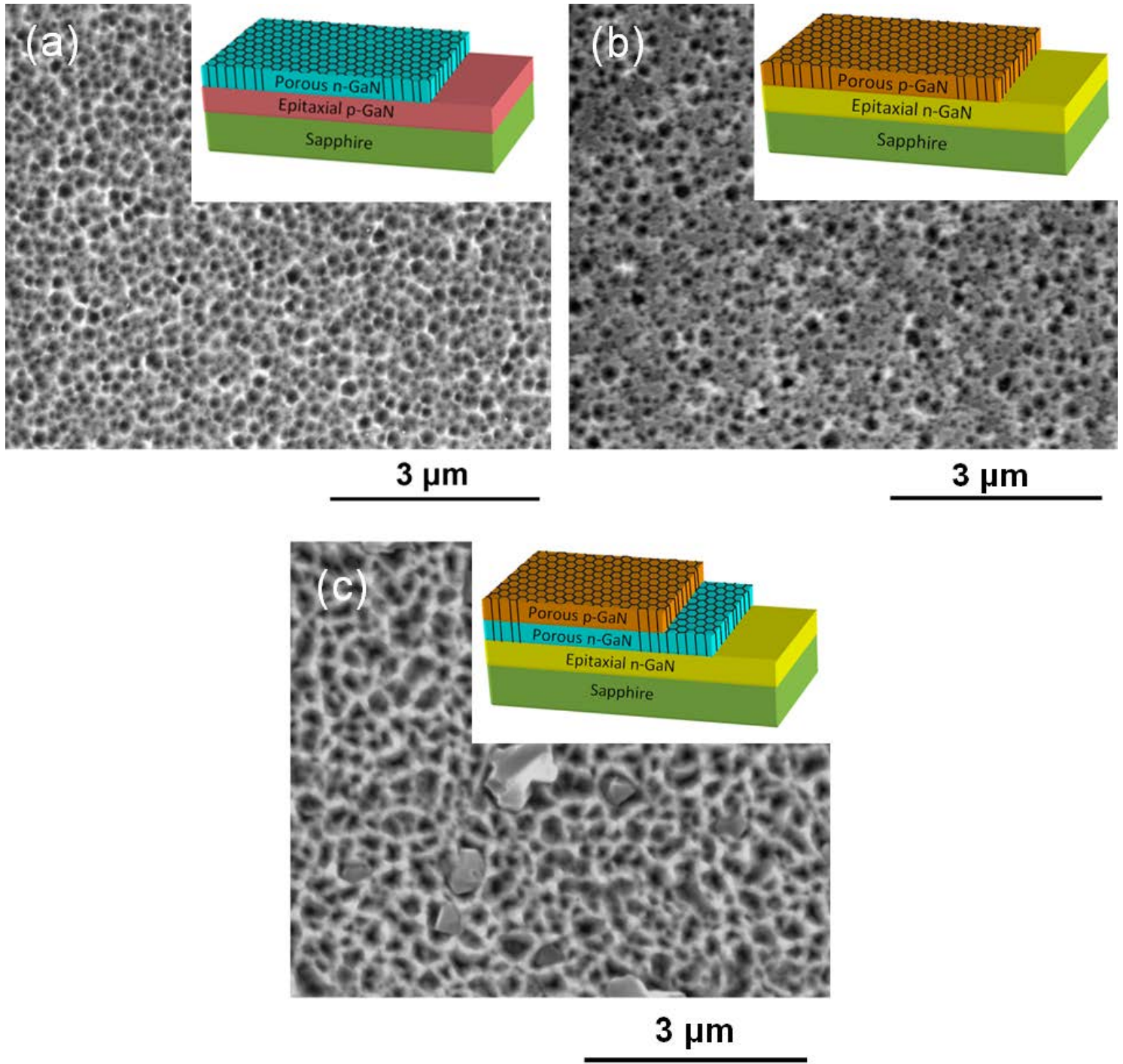

Figure 2. (a) Porous $n$-type GaN grown on non-porous epitaxial p-type GaN, (b) porous $p$-type GaN grown on non-porous epitaxial $n$-GaN, and (c) porous $p$-type GaN grown on porous $n$-type GaN corresponding to the fully porous diode. Insets show schemes of the structure of the partially porous diodes fabricated.

X-ray rocking curves corresponding to the (0004) reflection were used to analyze the influence of structural strain on porous GaN $p-n$ junction diodes, as shown in Figure 3. Figure 3(a) shows the rocking curves of the porous $n-p$ diode, defining the response of the epitaxial $p$ - and $n$-type GaN substrate for comparison, with FWHM of $0.428^{\circ}$ and $0.419^{\circ}$ for the non-porous and porous layers, respectively, indicating a good orientation of the porous material along the $\boldsymbol{c}$-crystallographic direction, also, it is observed that the crystalline quality of the porous layer is comparable to the non-porous film, or even better. The peak positions are $72.902^{\circ}$ and $73.082^{\circ}$ for the non-porous and porous layers, respectively, indicating a slight relaxation of the porous layer when compared to the non-porous one. The magnitude of the relaxation can be estimated according to the expression [12]: 
were $\boldsymbol{c}_{\text {strained }}$ and $\boldsymbol{c}_{\text {relaxed }}$ are the $\boldsymbol{c}$ crystallographic parameters of the non-porous $p$-type and porous $n$-type GaN layers, respectively, both calculated from the analysis of the rocking curves. Accordingly, a reduction of the strain perpendicular to the (0001) plane of $0.24 \%$ was estimated, which stems from the benefit of internal porosity (for details, see Table S1 of the Supporting Information).

Figure 3(b) shows the rocking curves of the porous $p$ - $n$ diode. The relaxation of the $p$-type porous layer is $0.46 \%$, almost twice that achieved in the porous $n$ - $p$ diode. In that case, FWHM is slightly higher for the porous layer, but of the same order as for the non-porous layer, indicating that the crystalline quality of these two films is similar. The relaxation of the porous $p$-type layer is larger than the one observeded for the porous $n$ type layer, which might be related to the wider size of its pores (see Figure 2(b)), giving additional compliance to the layer to accommodate on the substrate.

Finally, the fully porous GaN diode exhibits equal FWHM in their rocking curves for the two porous layers and of the same order to that of the non-porous $n$-type GaN film (see Figure 3(c) and Table S1). Thus, the crystalline quality of the three films is closely similar. The relaxation calculated for these porous layers is $0.04 \%$ for the $n$ type porous layer grown on the non-porous $n$-type GaN film, while that of the $p$-type porous layer grown on the porous $n$-type layer is $0.07 \%$. In both homo and heterojunctions, relaxed porous layers are epitaxially grown on either porous or continuous thin films. The minimum strain is found between the non-porous and the porous $n$-type GaN layers. 


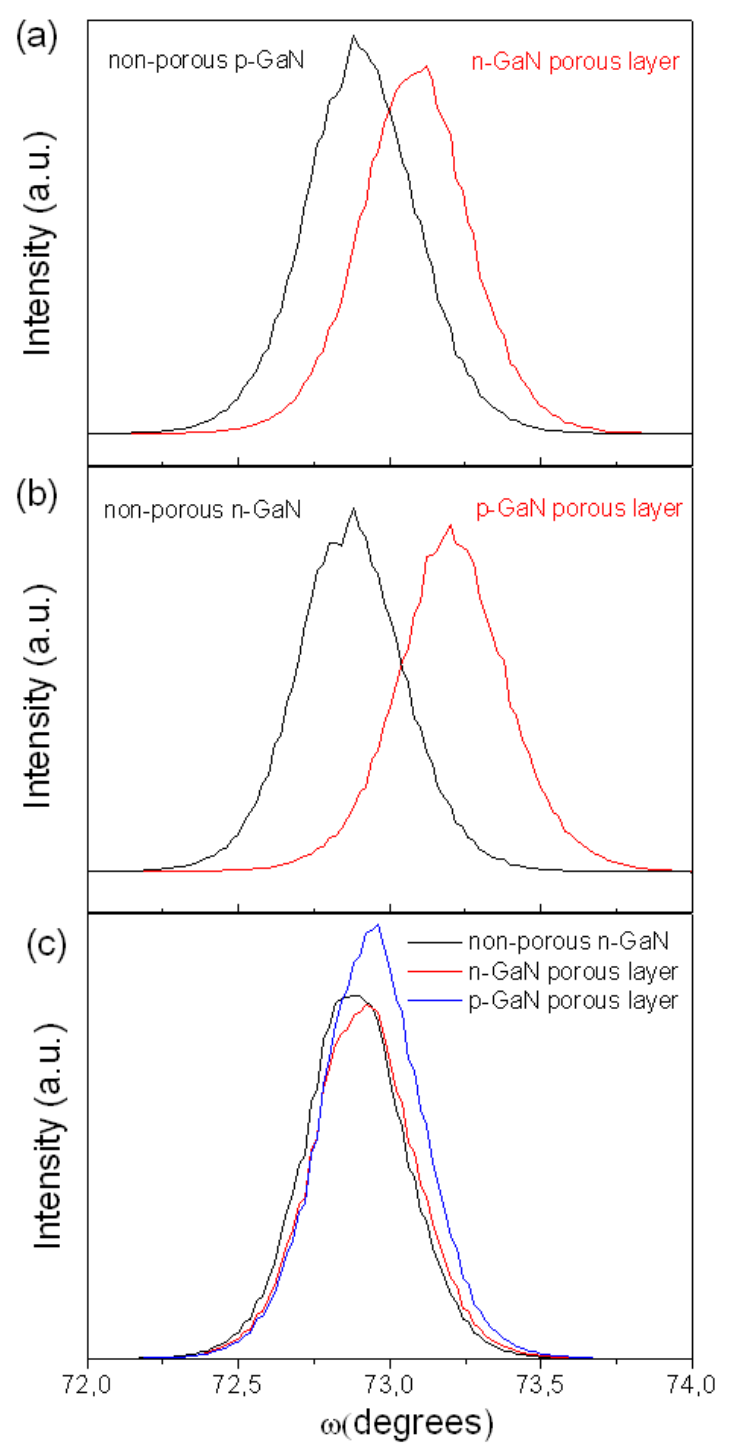

Figure 3. Rocking curves of (a) the porous $n$ - $p$, (b) porous $p-n$ and (c) fully porous diodes.

Figure 4 shows SEM and panchromatic cathodoluminescence (panCL) images, as well as CL spectra recorded for all the layers forming the fully porous $p$ - $n$ diode. The SEM image of the non-porous $n$-type GaN film shows a smooth surface, while the panCL image shows the typical granular luminescence pattern of $\mathrm{GaN}$, due to the distribution of clusters of dislocations in the material [13,14]. The CL spectrum recorded for this layer shows the near-band gap emission (NBE) located at $\sim 355.6 \mathrm{~nm}$ and a donor-acceptor pair (DAP) luminescence at $\sim 377 \mathrm{~nm}$, which characteristically results from point defects and/or impurities [15].

The panCL image of the porous $n$-type GaN layer shows a different distribution of the luminescence emission, that could be related to the porous morphology, even if there is not a full correlation between the pores revealed in the SEM image and the panchromatic image contrast (see Figure 4b). Tthe pores clearly seen in the SEM image, are not visible in the CL image, except for the larger ones, showing as dark contrast in the CL image. The spectrum recorded for this layer exhibits quite a broad NBE luminescence, corresponding to $n$-type $\mathrm{GaN}$, peaking at $\sim 362 \mathrm{~nm}$. A yellow luminescence (YL) band centered at $\sim 580 \mathrm{~nm}$, normally attributed to point defects (Ga vacancies) and/or impurities, such as oxygen and carbon [16] is also present. 
(a)
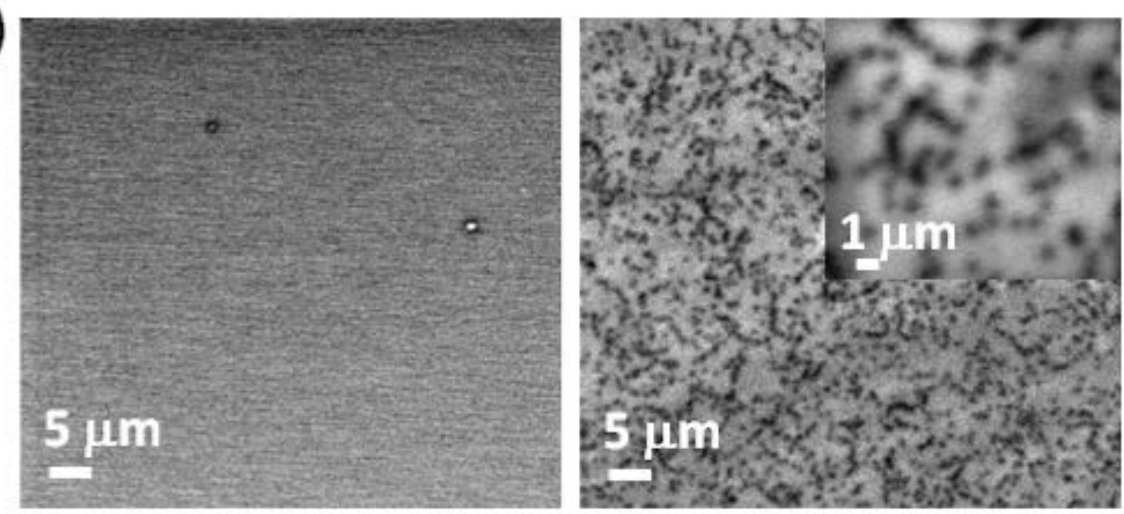

(b)
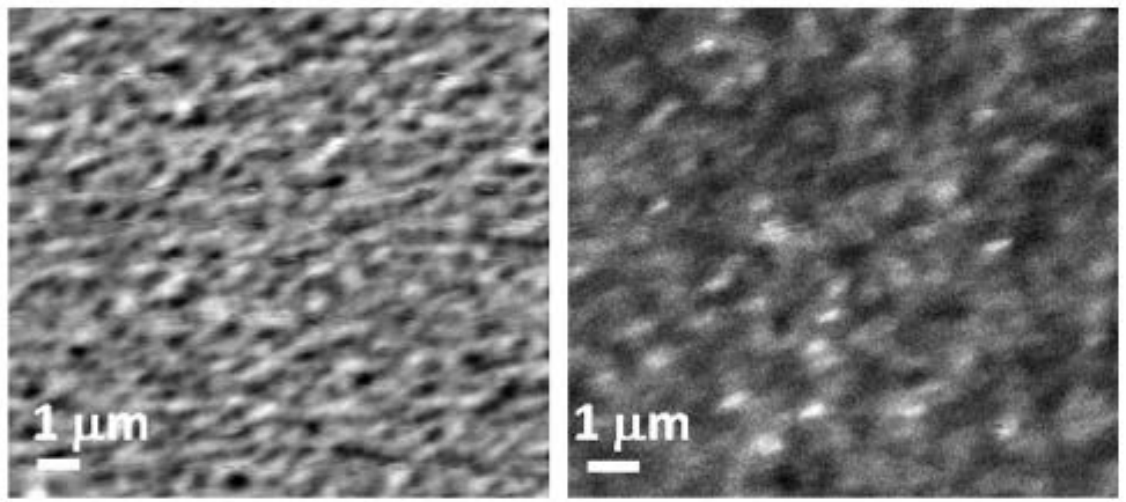

(c)
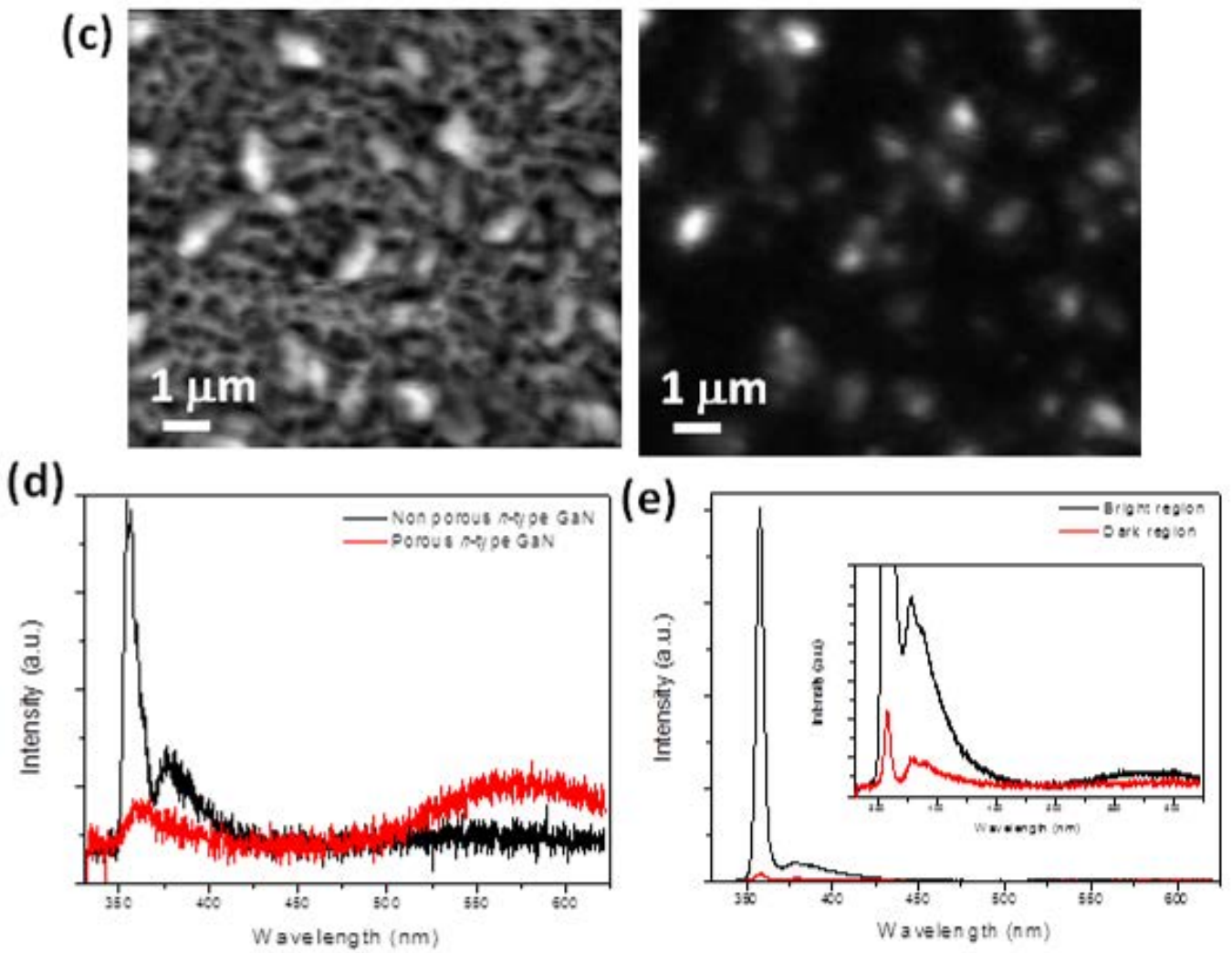

Figure 4: Top-view SEM and CL images taken in the same region of the sample of (a) non-porous $n$-type GaN film, (b) porous $n$-type GaN layer and (c) porous $p$-type GaN, corresponding to the fully porous $p$ - $n$ diode. Insets show magnified areas of the pictures. 
CL spectra recorded for (d) non-porous and porous $n$-type GaN, and (e) bright and dark areas observed in the panCL image shown in (c). Inset in the graph is a magnification of the same spectra to show the features observed in the two spectra.

Figure 4c shows the SEM and the corresponding panCL image of the top layer of the fully porous $p$ - $n$ diode (porous $p$-type $\mathrm{GaN}$ ). The pores are not visible in the panCL image, similar to what was observed in the porous $n$-type layer. High intensity regions correspond to porous GaN micron-size particles that grow on the top of porous layer, but with a different crystallographic orientation. The CL spectra shown in Figure 4e correspond to the high and low intensity emission regions observed in the panCL image, with the NBE of GaN at $\sim 358 \mathrm{~nm}$, the DAP band at $\sim 379 \mathrm{~nm}$ and a longitudinal optical (LO) phonon replica at $\sim 388 \mathrm{~nm}$. These features correspond to the typical CL spectrum of $p$-type GaN. However, the spectrum recorded in the high intensity region, corresponding to a GaN micron-size particle grown on the porous layer, has a much lower DAP intensity emission relative to the NBE emission, compared to the spectrum recorded in the low intensity region of the porous layer. This effect is likely related to a different $\mathrm{Mg}$ doping incorporation of the $\mathrm{GaN}$ porous layer and $\mathrm{GaN}$ micron-size particles [17] or to a different crystallographic orientation of the two regions [18], which also can be responsible for changes in the incorporation of the Mg impurities.

SEM and corresponding panCL cross-section images of the totally porous $p-n$ diode were recorded, as shown in Figures 5. The cross-sectional SEM micrograph clearly shows the non-porous GaN film and the porous GaN layers grown by CVD. In the panCL image, the dark lines observed in the non-porous $\mathrm{GaN}$ correspond to the threading dislocations crossing the substrate layer. According to the SEM image, the thickness of the porous GaN layers is $\sim 2.1 \mu \mathrm{m}$, which would correspond to the two porous layers, the Mg-doped porous $p$-type GaN and the undoped porous $n$-type GaN layer, each with a thickness of $\sim 1 \mu \mathrm{m}$. Although the interface between these two porous layers is difficult to appreciate in the SEM image, it appears quite clear in the panCL image, where porous $n$-type GaN appears dark while porous $p$-type GaN appears bright. This can be related to a higher concentration of charge carriers in the Mg-doped porous p-type GaN layer $\left(\sim 10^{18} \mathrm{~cm}^{-3}\right)$ when compared to the undoped porous $n$-type $\mathrm{GaN}$ $\left(\sim 10^{16} \mathrm{~cm}^{-3}\right)$, as previously consigned [9]. The thickness of the two porous layers is proved to be similar in the panCL image. Figure 5c shows the CL spectra recorded for the different layers in the cross section view. In the case of the non-porous $n$-type GaN film, a strong emission at $355 \mathrm{~nm}$ is observed similar to the one recorded in top-view (see Figure 4a). In the case of porous $p$-type GaN only the DAP emission related to the Mg doping is observed, with no emission from NBE, in contrast with that observed in top-view (see Figure 4e), indicative of the incorporation of substitutional $\mathrm{Mg}$ for an efficient $p$-type doping of this layer. Concerning the porous $n$-type $\mathrm{GaN}$ layer, nearly no CL signal was detected, in agreement with the low intensity detected in the top-view configuration (see Figure 4d). The appearance of the yellow band, not observed for the non-porous substrate, confirms that the cross-sectional spectrum corresponds to the porous layer. 


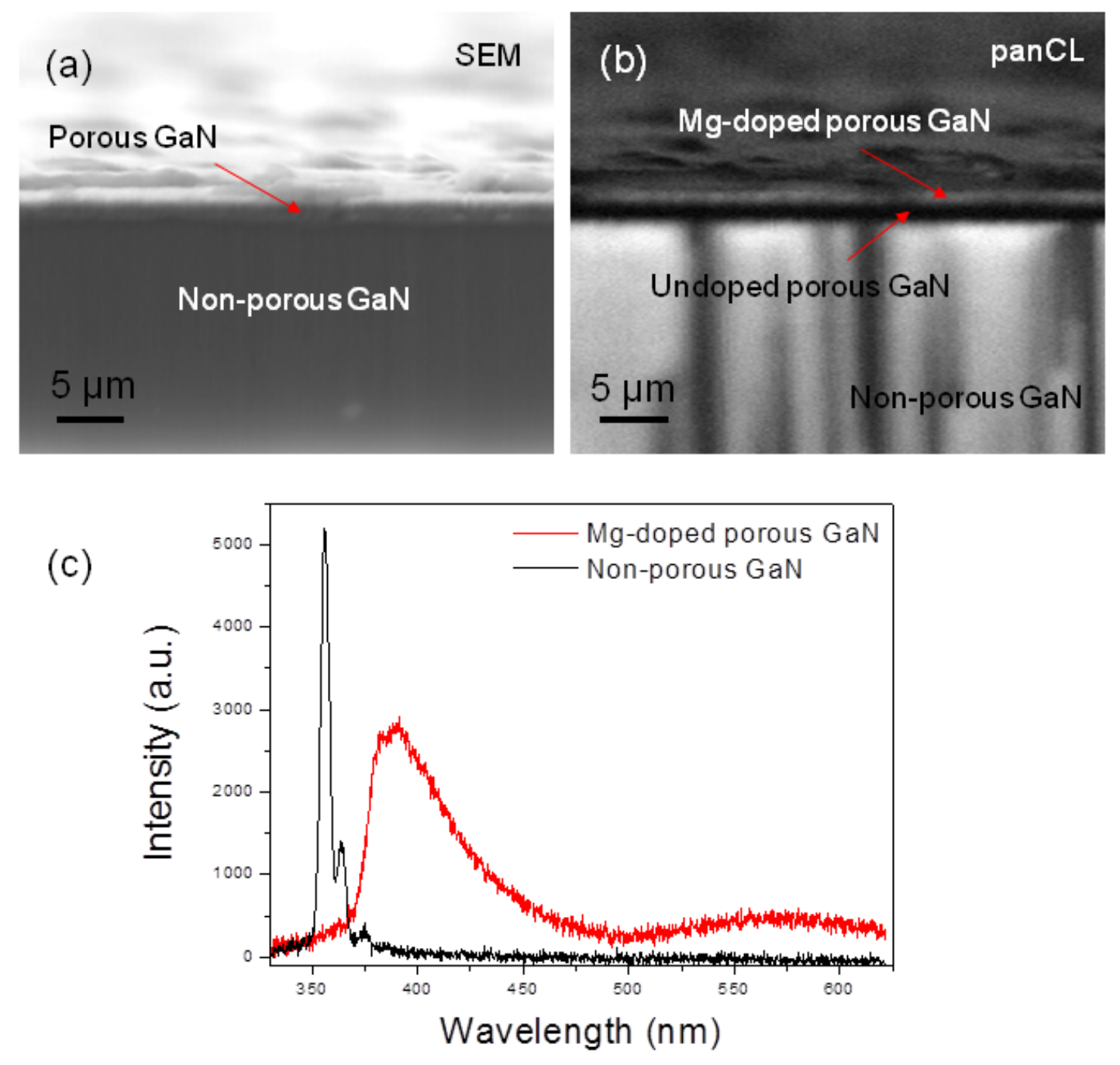

Figure 5: (a) Croos sectional SEM and (b) corresponding panCL images of the fully porous GaN diode. (c) CL spectra recorded at bright and dark regions observed in pannel (b).

Photoluminescence (PL) measurements in top view were also performed on both Mg-doped porous $\mathrm{GaN}$ and undoped porous $\mathrm{GaN}$ layers corresponding to the totally porous GaN p- $n$ junctions at different temperatures from $11 \mathrm{~K}$ to $300 \mathrm{~K}$ at a constant laser power density. Figure 6a shows PL spectra for undoped porous GaN. It exhibits the expected band-edge luminescence with a Varshni-like temperature redshift from $3.43 \mathrm{eV}$ at $300 \mathrm{~K}$ to $3.46 \mathrm{eV}$ at $11 \mathrm{~K}$ and a narrow FWHM of $12 \mathrm{meV}$ at $11 \mathrm{~K}$. Broad emission near the band gap typically results from the density of states tailing due to randomly distributed impurities. This broad emission near-band gap and band-edge emission equilibrate in intensity at $100 \mathrm{~K}$ and the broad emission near-band gap slightly dominates at $120 \mathrm{~K}$. However, the band-edge emission dominates at other temperatures.

Figure $6 \mathrm{~b}$ shows PL spectra for the $\mathrm{Mg}$ doped porous $\mathrm{GaN}$. The room temperature band-edge emission appears at lower energy $(3.41 \mathrm{eV})$ when compared to the undoped porous $\mathrm{GaN}$. At temperatures down to $11 \mathrm{~K}$, however, this emission is blue-shifted and located at a value of $3.46 \mathrm{eV}$ with a narrow FWHM of $\sim 5 \mathrm{meV}$. The band gap emission in both, undoped and $\mathrm{Mg}$ doped $\mathrm{GaN}$, is over an order of magnitude more intense at 11 
$\mathrm{K}$ as compared to room temperature. We observe some structure in the high energy side, at $\sim 3.5 \mathrm{eV}$, probably associated with a bound exciton. As the $\mathrm{GaN}$ is $\mathrm{Mg}$ doped and no Si nor shallow $\mathrm{O}$ donor-bound excitons are expected to be incorporated in the samples according to the synthesis conditions, we attribute this weak emission to excitons bound to $\mathrm{Mg}$ acceptors. The band-edge luminescence is followed by the DAP luminescence, located at 3.27 and $3.28 \mathrm{eV}$ at 150 and $11 \mathrm{~K}$, respectively. The DAP emission disappears at high temperatures. As expected from $\mathrm{Mg}$ acceptors in doped $\mathrm{GaN}$, we find the LO phonon replicas at 3.18 and $3.19 \mathrm{eV}$ at 150 and $11 \mathrm{~K}$ respectively, with several harmonics observable at $11 \mathrm{~K}$, indicating the good electronic quality of the $p$-type porous GaN layer obtained by CVD [19]. Another broad band at $420 \mathrm{~nm}$ (blue band) is also clearly observed at $300 \mathrm{~K}$, disappearing as the temperature decreases ( $200 \mathrm{~K})$. This type of emission has been previously observed and attributed to the formation of a Mg complex or some native defect level.

YL in $n$-type GaN is often attributed to Ga vacancies, and impurities, such as oxygen and carbon [16], although it is not likely that these impurities are present in the samples through the growth process used. YL is also present in Mg-doped p-type porous GaN and can arise from a transition between the conduction band or shallow donors [20]. The YL has weak temperature dependence and is not found to vary considerably due to $\mathrm{Mg}$ incorporation into the lattice when compared to the undoped sample.
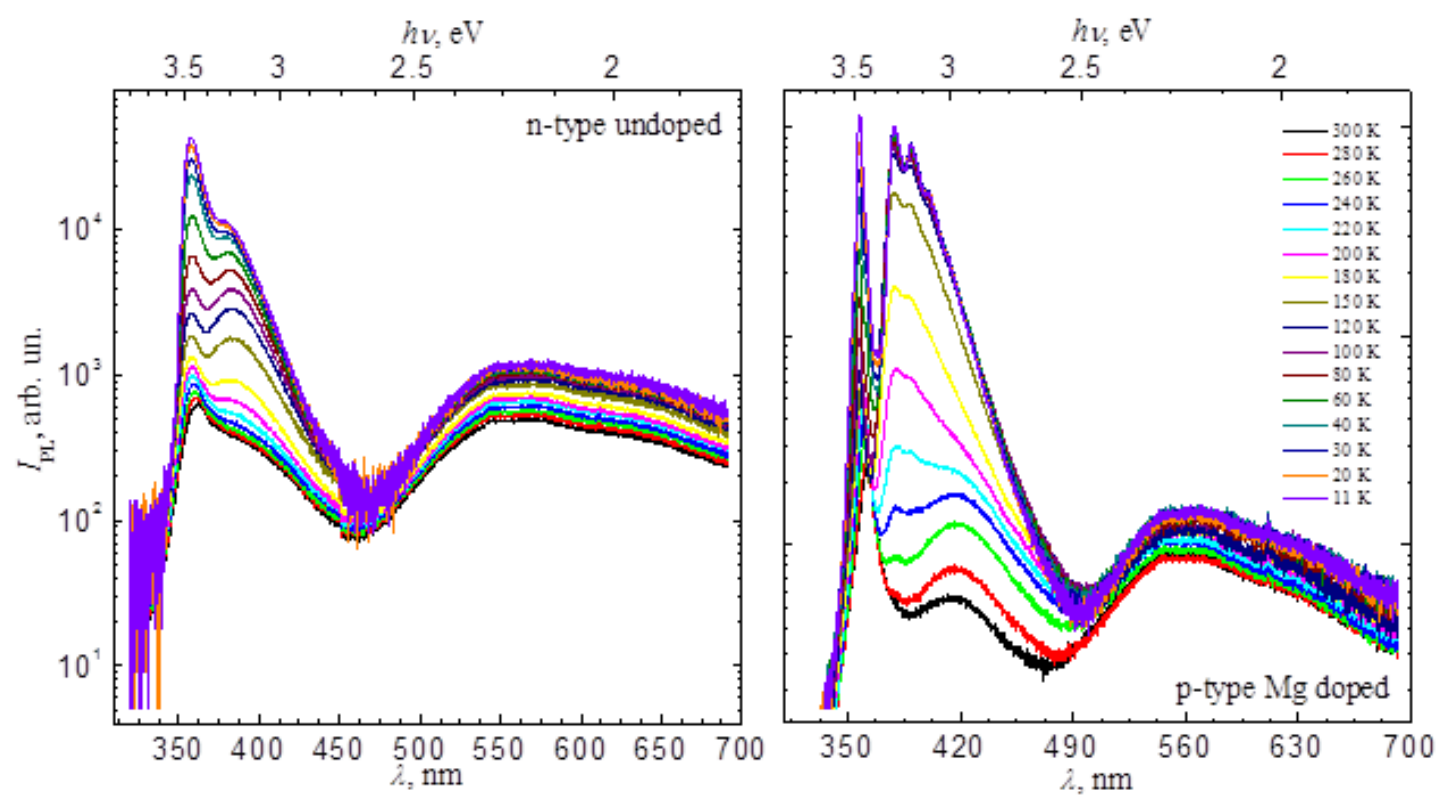

Figure 6. Temperature-dependent PL spectra of undoped (a) and Mg-doped GaN (b) layers corresponding to the fully porous $\mathrm{GaN}$ p-n junction $\left(\lambda_{\mathrm{ex}}=325 \mathrm{~nm}\right)$.

Electrical characterization of the three types of porous GaN diodes is shown in Figure 7. Diodes comprising non-porous and porous $\mathrm{GaN}$, and also fully porous $p-n$ GaN junction exhibited characteristic I-V curves with strong rectification. Figure $7 \mathrm{a}$ shows the I-V curve of the porous $n-p$ diode. The high bias resistance turn-over of the porous $n$-type $\mathrm{GaN}$ occurs just above $1 \mathrm{~V}$. When the non-porous $p$-type $\mathrm{GaN}$ is positively biased, current flow through the $p-n$ junction varies exponentially. The barrier to the exponential current increase is found to be much lower than the expected GaN 
diode response, i.e. $E_{\mathrm{g}} / q$. The turn-on voltage for this $p$ - $n$ junction lies in the range $E_{\mathrm{g}} / 4 q-E_{\mathrm{g}} / 2 q$, i.e. $0.5-0.68 \mathrm{~V}$. In the case of the porous $p$ - $n$, and the fully porous $\mathrm{GaN}$ diode the turn-on voltage is found to be even lower, in the range $E_{\mathrm{g}} / 4 q-E_{\mathrm{g}} / 6 q$ (see Figure $7 \mathrm{~b}$ and $7 \mathrm{c}$ ). In all cases the diode behavior is confirmed and low reverse bias leakage currents were found for these porous GaN structures. Moreover, the current at a fixed value of the forward voltage in the fully porous diode is found to be three orders of magnitude higher than that measured in the partially porous diodes, indicating a low resistivity for porous GaN layers and its promising perspectives for LEDs applications.

The electron density for unintentionally doped porous $n$-type GaN is estimated to be $1.6 \times 10^{16} \mathrm{~cm}^{3}$ [9a]. The effective hole density for porous $p$-type $\mathrm{GaN}$ at $300 \mathrm{~K}$ is estimated using the effective hole and electron masses of wurzite $\mathrm{GaN}$ to be $\sim 9 \times 10^{18}$ $\mathrm{cm}^{-3}$ [9b]. Taking into account the values of donor and acceptor densities of non-porous $n$-GaN and $p$-GaN of $N_{D}=10^{19} \mathrm{~cm}^{-3}$ and $N_{A}=10^{18} \mathrm{~cm}^{-3}$, respectively, and the corresponding values mentioned above for porous $n-\mathrm{GaN}$ and $p-\mathrm{GaN}$, we can estimate $V_{B}$ for the porous $n-p$ diode as $0.72 \mathrm{~V}$, for the porous $p$ - $n$ diode as $0.9 \mathrm{~V}$, and for the totally porous diode as $0.71 \mathrm{~V}$.

Based on these measurements we should mention some important aspects for porous GaN $p$ - $n$ junctions. Microscopic characterization confirms well-defined interface between the porous and the non-porous GaN layers (see Figure 5) and thus no significant tunnelling barrier exists either at the GaN-GaN interface or on the surface of the GaN / Ohmic contact. Also, microscopic characterization excludes any interfacial contaminants that would alter the effective barrier. The measurements were repeated from different points on several separate $p$ - $n$ junction diodes and they exhibited similar $\mathrm{I}-\mathrm{V}$ response, which augers well for high surface area diodes and devices form completely porous $p-n$ junctions over large areas [21]. 


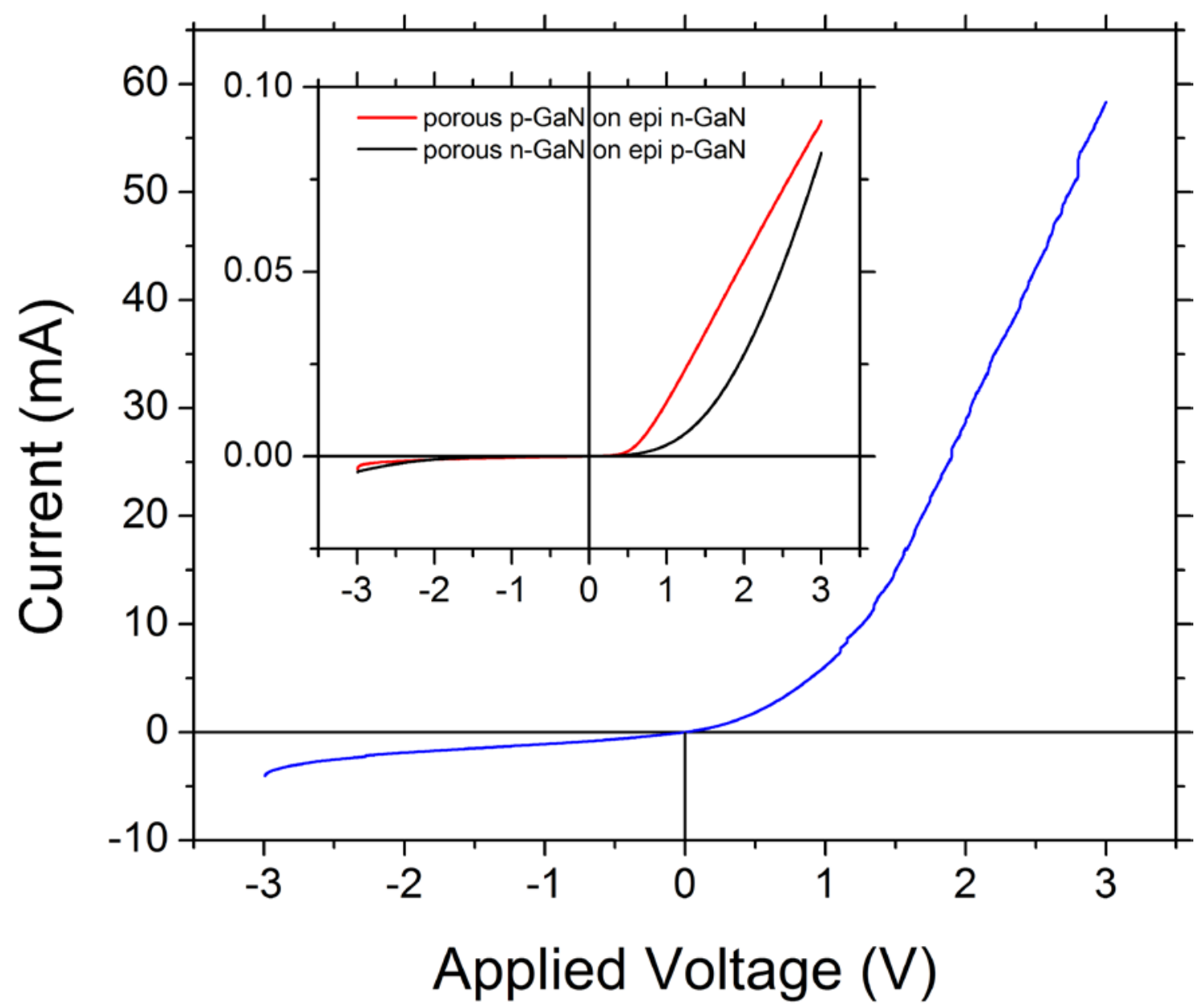

Figure 7. I-V curves of the porous $n-p$ diode (a), porous $p$ - $n$ diode and totally porous diode (c).

Figure 8 shows the I-V curves recorded for the porous $n$ - $p$ diode at different temperatures ranging from $298 \mathrm{~K}$ to $673 \mathrm{~K}$, confirming that we are dealing with a rectifying diode in all $p$ - $n$ junctions. The forward and reverse bias currents of the diode increase with the temperature, thus accounting for an increase of the number of charge carriers across the barrier height. The turn-on voltage for this junction is characteristically $\sim E_{\mathrm{g}} / 4 q-E_{\mathrm{g}} / 2 q$ at room temperature. With the increase of temperature, this low knee voltage is reduced even further consistent with a thermionic process with greater carrier velocities at higher temperatures. At the highest temperatures the high bias resistance turn-over of the porous $n$ - $p$ diode occurs at very low potentials, but the built-in potential $\mathrm{V}_{\mathrm{bi}}=(k T / q) \ln \left(\mathrm{N}_{\mathrm{A}} \mathrm{N}_{\mathrm{D}} / n_{\mathrm{i}}{ }^{2}\right)$ is overcome through tunneling. At all temperatures (at least to $673 \mathrm{~K}$ ) the rectifying characteristics of the porous $\mathrm{GaN}$ diodes are preserved, and no breakdown voltage was observed even at $10 \mathrm{~V}$ at $673 \mathrm{~K}$. 


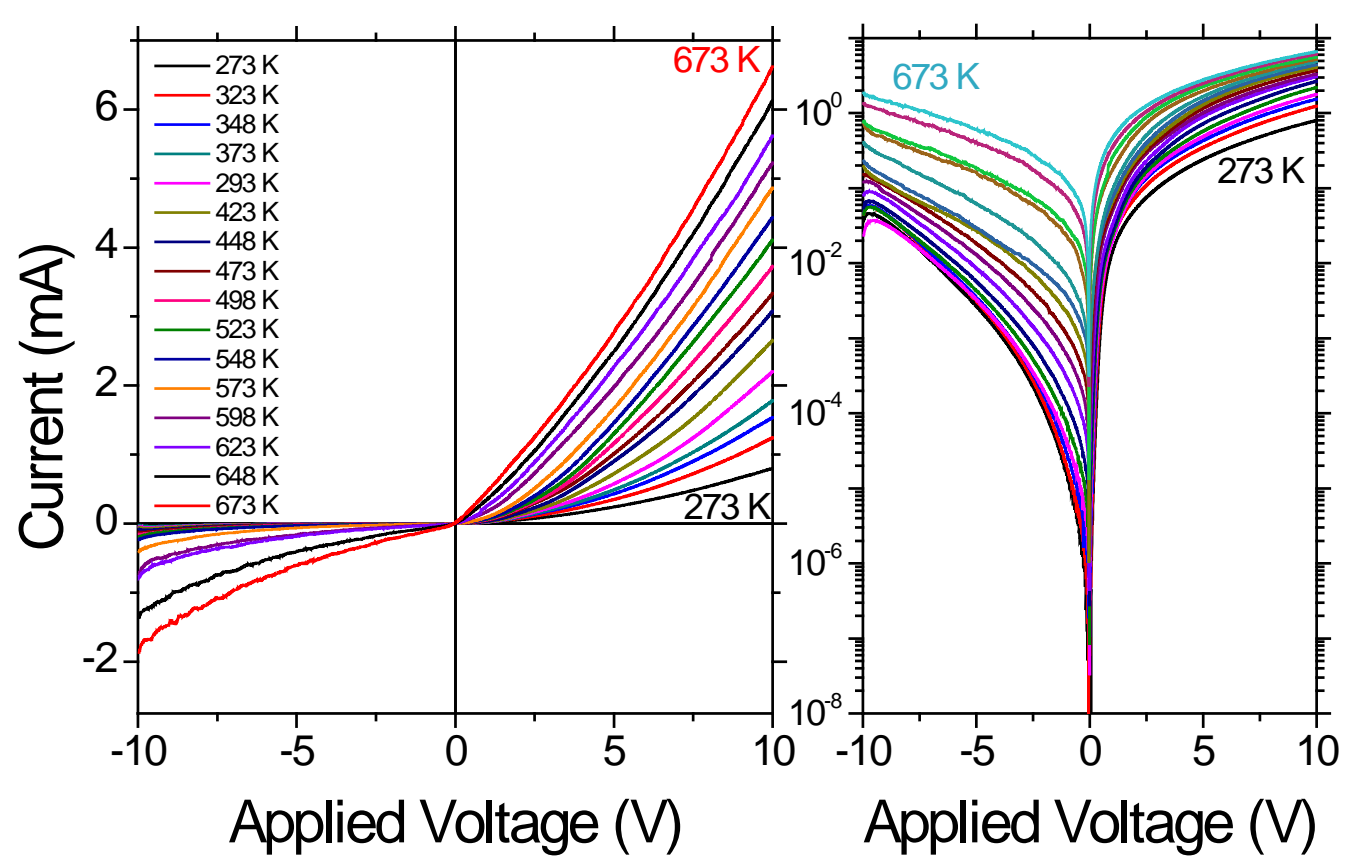

Figure 8. I-V curve of the porous $n-p$ GaN diode recorded at $\mathrm{T}=298-673 \mathrm{~K}$.

The fully porous GaN $p$ - $n$ junction diodes, and those comprising porous $n$ - or $p$ type GaN on non-porous epilayers, all exhibited stable rectification. Figure 9 shows current rectification achieved at reverse bias and forward bias (>the knee voltage) recorded for the fully porous $p$ - $n$ diode (see Figure S2 of the Supplementary Information for current rectification in partially porous diodes) at a bias polarity switch frequency of $0.1 \mathrm{~Hz}$ at room temperature. The I-t characteristics recorded at $\pm 0.4 \mathrm{~V}$ does not show clear rectification behavior, since this voltage is below or on the limit of the turn-on voltage of the porous GaN diodes. At $\pm 1 \mathrm{~V}, \pm 2 \mathrm{~V}, \pm 3 \mathrm{~V}$ and $\pm 5 \mathrm{~V}$ voltages all diodes demonstrate rectifying behavior. The highest leakage voltage and the lowest rectification ratio is observed at $\pm 5 \mathrm{~V}$. Also, porous $\mathrm{GaN}$ diodes demonstrate very stable values of current with time at both, forward and reverse bias. The stability confirms that for porous $p-n$ junctions using a single porous layer deposited on an epitaxially continuous GaN film, or from a porous layer grown on another porous layer, a remarkable stability in rectification is maintained. Porous GaN films can exhibit random porosity (compared to arrays of nanostructures), but their ease of deposition over large area without dominating leakage currents is promising for wide bandgap applications including sensors. 


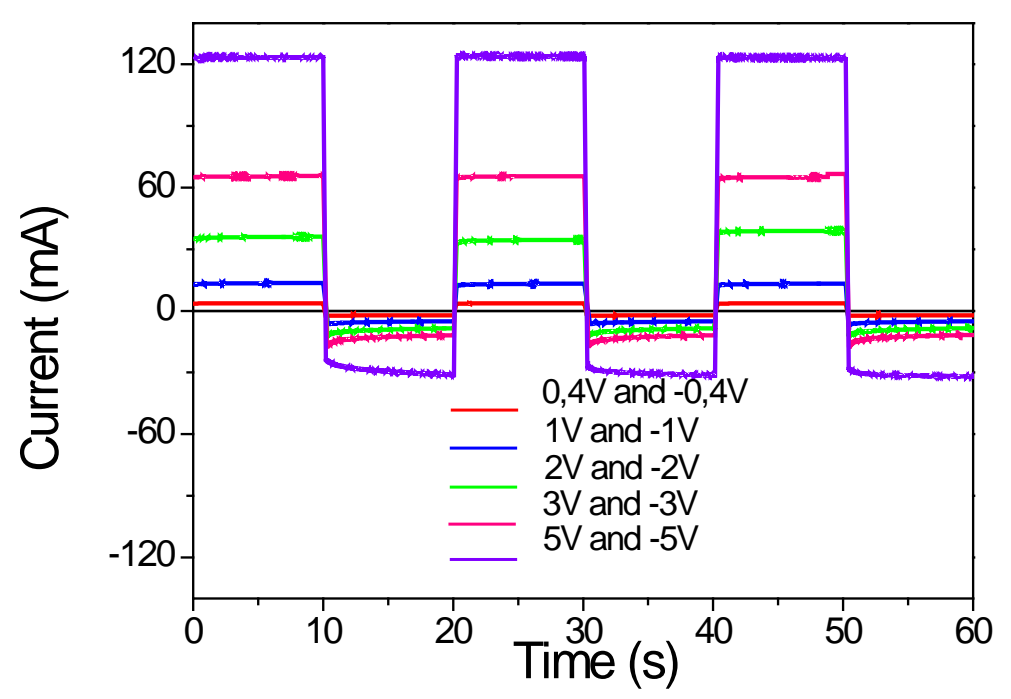

Figure 9. Rectifying characteristics of the fully porous diode shown at different voltages.

Taken as a whole, the investigation presented here confirms that completely porous $p$ - $n$ junction of porous $n$-type and porous $p$-type GaN can be grown over large areas by CVD. The structural (X-ray diffraction) and optical characteristics confirm high quality GaN growth for porous layers when compared with non-porous epitaxially grown GaN, even when overgrown with opposite conduction type porous layer to form porous/non-porous $p$ - $n$ junction diodes. We intend to extend this investigation to other III-N materials such as InN and AlN to span the visible spectrum, and as a route towards porous, graded index III-N materials as a basis for white light LEDs incorporating other colour centers, or for reducing reflection losses and narrowing the output light cone for improved LED external quantum efficiencies.

\section{Experimental Section}

Fabrication of partially and totally porous GaN diodes. Nanoporous GaN thin layers were grown on commercial non-porous $\mathrm{GaN}$ thin films with complementary electrical conductivity produced on sapphire (0001) substrates. The porous films were grown by the direct reaction of metallic Ga with ammonia in a tubular CVD system, using gallium metal (99.999\%) and ammonia (99.99\%), as $\mathrm{Ga}$ and $\mathrm{N}$ sources, respectively. To produce porous $p$-type $\mathrm{GaN}, \mathrm{Mg}$ was introduced in the furnace in the form of magnesium nitride (99.95\%), placing it $4 \mathrm{~cm}$ up-stream of the Ga source. The substrate was placed $1.7 \mathrm{~cm}$ above the Ga source. Prior to their introduction in the furnace, the substrates were cleaned with ethanol. When the substrate and the Ga and $\mathrm{Mg}$ precursors were introduced in the furnace, the quartz tube of the furnace was degassed to a vacuum pressure of $1 \times 10^{-2}$ Torr. Ammonia was then introduced through a mass-flow controller at a flow rate of $75 \mathrm{sccm}$, while the pressure was set at 15 Torr and the furnace was heated up to the reaction temperature of $1203 \mathrm{~K}$. Then, the furnace was kept at constant temperature for 60 min under constant $\mathrm{NH}_{3}$ flow and pressure, while the chemical reaction took place. When the reaction was finished, the furnace was 
cooled down to room temperature while the ammonia flow was stopped, thus the pressure of the system dropped to $1 \times 10^{-2}$ Torr. After growth, the samples containing porous Mg-doped $\mathrm{GaN}$ were annealed at $973 \mathrm{~K}$ in $\mathrm{N}_{2}$ atmosphere for $20 \mathrm{~min}$ in order to break Mg-H complexes and activate the $p$-type conductivity in porous $\mathrm{GaN}$.

Morphological and structural characterization of porous GaN. Nanoporous GaN layers deposited on GaN (0001) substrates were characterized morphologically using a JEOL JSM 6400 scanning electron microscope (SEM). Rocking curves were recorded using a Bruker-AXS D8-Discover diffractometer equipped with parallel incident beam (Göbel mirror), a vertical $\theta-\theta$ goniometer, a XYZ motorized stage and a General Area Diffraction Detection System (GADDS). Samples were placed directly on the sample holder and the area of interest was selected with the aid of a video-laser focusing system. The X-ray diffractometer was operated at $40 \mathrm{kV}$ and $40 \mathrm{~mA}$ to generate $\mathrm{Cu} \mathrm{K \alpha}$ radiation. The GADDS detector was a HI-STAR (multiwire proportional counter of $30 \times 30 \mathrm{~cm}^{2}$ with $1024 \times 1024$ pixels). The rocking curves covered an omega angle of $6^{\circ}$, collected through 120 frames at a step size of $0.05^{\circ}$ and $15 \mathrm{~s}$ of exposition time per frame.

Cathodoluminescence and photoluminescence characterization. CL measurements were carried out in monoCL2 system from Gatan attached to a field emission scanning electron microscope (FESEM) (Carl Zeiss-LEO 1500) operating at $10 \mathrm{kV}$. The measurements were carried out at $80 \mathrm{~K}$. The detection for panCL images was done with a photomultiplier (MPT), and the CL spectra were recorded with a Peltier cooled charge coupled device (CCD) detector. PL was collected exciting the samples with the $244 \mathrm{~nm}$ line (second harmonic of $488 \mathrm{~nm}$ line) from a cw Ar-ion laser with a power density of 2 $\mathrm{W} / \mathrm{cm}^{2}$. PL spectra were acquired using a Horiba iHR320 spectrometer equipped with a Synapse CCD matrix. Samples were placed in an evacuated chamber of a Janis closedcycle helium cryostat for PL measurements at different temperatures.

Electrical characterization. Two point electrical measurements of partially and totally porous GaN diodes were conducted using In/Ga liquid eutectic contacts and a Biologic SP-50 potentiostat. An In/Ga eutectic droplet was placed on top of the porous $\mathrm{GaN}$ material with the other contact placed directly on the non-porous epitaxial $n$ - or $p$ type GaN, in the case of the partially porous diodes, or on the porous n-type GaN layer in the case of the totally porous diode. Linear voltage sweeps were obtained in the range between $-3 \mathrm{~V}$ and $3 \mathrm{~V}$ with a $50 \mathrm{mV} / \mathrm{s}$ sweep rate and of $-10 \mathrm{~V}$ and $10 \mathrm{~V}$ with a $50 \mathrm{mV} / \mathrm{s}$ sweep rate for temperature-dependent measurements. Diode rectification measurements were conducted in the range between $\pm 0.4 \mathrm{~V}$ and $\pm 5 \mathrm{~V}$ voltages for forward and reverse bias. The measurements were repeated with contacts on various points of each sample to ensure repeatability.

\section{Acknowledgements}

This project was supported by the EU Framework 7 under Project No. FP7-SPA2010-263044, the Spanish Government under Projects No. MAT2011-29255-C02-02, TEC2010-21574-C02-02, MAT2010-20441-C02-01-02, MAT-2010-16116 and MAT2010-20441-C02-01-02, by the Catalan Authority under Project No. 2009SGR235 and by the "Conserjería de Educación de la Junta de Castilla y León" under Project No. VA166A11-2 and VA293U13. This work was also supported by the Irish Research 
Council New Foundations Award. O.V. Bilousov is supported by Generalitat de Catalunya through the fellowship 2013FI_B2 00108.

\section{References}

1. (a) Anual Energy Review 2011, U.S. Energy Information Administration, DOE/EIA-0384 (2011); (b) S. Nakamura, S. Pearton, G. Fasol, G. The blue laser diode: The complete story; Springer-Verlag: Berlin, 2000.

2. (a) M. Hwang, W. H. Hung, and H. L. Hwang, IEEE Photon. Technol. Lett. 2008, 20, 608; (b) J.J. Wierer, A. David, M.M. Megens, Nature Photon. 2009, 3, 163; (c) T.K. Kim, S.H. Kim, S.S. Yang, J.K. Son, K.H. Lee, Y.G. Hong, K.H. Shim, J.W. Yang, K.Y. Lim, S.J. Bae, G.M. Yang, Appl. Phys. Lett. 2009, 94, 161107; (d) C.H. Lee, J. Yoo, Y.J. Hong, J. Cho, Y.J. Kim, S.R. Jeon, J.H. Baek, G.C. Yi, Appl. Phys. Lett. 2009, 94, 213101; (e) D.S. Liu, T.W. Lin, B.W. Huang, F.S. Juang, P.H. Lei, C.Z. Hu, Appl. Phys. Lett. 2009, 94, 143502; (f) S.W. Ryu, J. Park, J.K. Oh, D.H. Long, K.W. Kwon, Y.H. Kim, J.K. Lee, J.H. Kim, Adv. Funct. Mater. 2009, 19, 1650; (g) M.H. Lo, P.M. Tu, C.H. Wang, C.W. Hung, S.C. Hsu, Y.J. Cheng, H.C. Kuo, H.W. Zan, S.C. Wang, C.Y. Chang, S.C. Huang, Appl. Phys. Lett. 2009, 95, 041109; (h) Y.M. Song, E.S. Choi, G.C. Park, C.Y. Park, S.J. Jang, Y.T. Lee, Appl. Phys. Lett. 2010, 97, 093110; (i) C.Y. Cho, S.E. Kang, K.S. Kim, S.J. Lee, Y.S. Choi, S.H. Han, G.Y. Jung, S.J. Park, Appl. Phys. Lett. 2010, 96, 181110; (j) S.Chhajed, W. Lee, J. Cho, E.F. Schubert, J.K. Kim, Appl. Phys. Lett. 2011, 98, 071102; (k) X.X. Fu, B. Zhang, X.N. Kang, J.J. Deng, C. Xiong, T. Dai, X.Z. Jiang, T.J. Yu, Z.Z. Chen, G.Y. Zhang, Opt. Exp. 2011, 19, A1104

3. (a) A.H.D. Graham, C.R. Bowen, J. Robbins, G. Lalev, F. Marken, J. Taylor, Sens. Actuators B 2010, 147, 697; (b) D. Valerini, A. Creti, A.P. Caricato, M. Lomascolo, R. Rella, M. Martino, Sens. Actuators B 2010, 145, 167; (c) S.J. Pearton, F. Ren, Adv. Mater. 2000, 12, 1571

4. Z. Fan, H. Razavi, J.W. Do, A. Moriwaki, O. Ergen, Y.L. Chueh, P.W. Leu, J.C. Ho, T. Takahashi, L.A. Reichertz, S. Neale, K. Yu, M. Wu, J.A. Ager, A. Javey, Nat. Mater. 2009, 8, 648

5. (a) E.J. Anglin, L. Cheng, W.R. Freeman, M.J. Sailor, Adv. Drug Delivery Rev. 2008, 60, 1266; (b) G. Jiménez-Cadena, J. Riu, F.X. Rius, Analyst 2007, 132, 1083

6. N. Bacci, A. Diligenti, G. Barillaro, J. Appl. Phys. 2011, 110, 036106

7. W. Sun, N.P. Kherani, K.D. Hirschmann, L.L. Gadeken, P.M. Fauchet, Adv. Mater. 2005, 17, 1230.

8. (a) J.J. Carvajal, J.C. Rojo, Cryst. Growth Des. 2009, 9, 320; (b) J.J. Carvajal, O.V. Bilousov, D. Drouin, M. Aguiló, F. Díaz, J.C. Rojo, Microsc. Microanal. 2012, 18, 905.

9. (a) O.V. Bilousov, J. J. Carvajal, D. Drouin, X. Mateos, F. Díaz, M. Aguiló and C. O’Dwyer. ACS Appl. Mater. Interfaces 2012, 4, 6927; (b) O. V. Bilousov, H. 
Geaney, J. J. Carvajal, V. Z. Zubialevich, P. J. Parbrook, A. Giguere, D. Drouin, F. Diaz, M. Aguilo and C. O’Dwyer, Appl. Phys. Lett. 2013, 103, 112103

10. W. Götz, N.M. Johnson, J. Walker, D.P. Bour, R.A. Street, Appl. Phys. Lett. 1996, 68, 667

11. S.J. Pearton, R.G. Wilson, J.M. Zavada, J. Han, R.J. Shul, Appl. Phys. Lett. 1998, 73, 1877

12. J.O. Song, J.S. Ha, T.Y. Seong, IEEE Electron. Dev. Lett. 2010, 57, 42.

13. S. Dassonneville, S. A. Amorkane, B. Sieber, J.L. Farvacque, B. Beaumont, P. Gibart, J. Appl. Phys. 2001, 89, 3736

14. X.L. Sun, S.H. Goss, L.J. Brillson, D.C. Look, R.J. Molnar, J. Appl. Phys. 2002, 91, 6729

15. M. Herrera Zaldivar, P. Fernández, J. Piqueras, J. Appl. Phys. 1998, 83, 462.

16. J. Neugebauer, C.G. Van de Walle, Appl. Phys. Lett. 1996, 69, 503

17. J.M. Myoung, K.H. Shim, S. Kim, Jap. J. Appl. Phys. Part 1 2001, 40, 476

18. F. Bertram, J. Christen, M. Schmidt, M. Topf, S. Koymov, S. Fischer, B. Meyer, Mater. Sci. Eng. B 1997, 50, 165.

19. L. Eckey, J. C. Holst, P. Maxim, R. Heitz, A. Hoffmann, I. Broser, B.Meyer, C. Wetzel, E. Mokhov, P. Baranov, Appl. Phys. Lett. 1996, 68, 415.

20. O Gelhausen, HN Klein, MR Phillips, EM Goldys, Appl. Phys. Lett. 2003, 83, 3293.

21. (a) Y. Huang, X. Duan, Y. Cui, C,M, Lieber, Nano Lett. 2002, 2, 101-104; (b) X. Duan, C.M. Lieber, J. Am. Chem. Soc. 2000, 122, 188 